\title{
Importance of Orbital Spectroscopy on Venus
}

Primary author: Joern Helbert

Phone number: $\quad+493067055319$

Institution: $\quad$ DLR

Email: $\quad$ Joern.Helbert@dlr.de

\section{Co-authors:}

M. Darby Dyar (PSI), David Kappel (DLR), Alessandro Maturilli (DLR), Nils Mueller (DLR)

\section{Co-signers:}

Giulia Alemanno (DLR), Jeff Andrews-Hanna (Univ. of Arizona), Pat Beauchamp (JPL), Giles

Berger (CNRS-Univ. P. Sabatier), Doris Breuer (DLR), Jeremy Brossier (Wesleyan University), Debra L. Buczkowski (APL), Paul K. Byrne (NCSU), Glyn Collinson (Goddard), Mario

D’Amore (DLR), Elizabeth Frank (First Mode), Martha Gilmore (Wesleyan Univ.), Candace

Gray (New Mexico State Univ./Apache Point Observatory), Scott Hensley (JPL), Robert Herrick (Univ. Alaska), Devanshu Jha (IAAC), Lauren Jozwiak (APL), Walter Kieffer (LPI), Siddharth Krishnamoorthy (JPL), Luciano Iess (Univ. La Sapienza), Noam Izenberg (APL), Kandis-Lea Jessup (SwRI), Yeon Joo Lee (Technische Univ. Berlin), Liming Li (Univ. of Houston), Emmanuel Marcq (LATMOS/IPSL/UVSQ), Phillipa J. Mason (Imperial College), Ryan McCabe (Hampton Univ.), Joe O'Rourke (ASU), Ana-Catalina Plesa (DLR), Takehiko Satoh (JAXA), Suzanne Smrekar (JPL), Claudia Stangarone (DLR), Joann Stock (Caltech), Timothy N. Titus (USGS Flagstaff), Allan Treiman (LPI), Constantine Tsang (SwRI), Indhu Varatharajan (DLR), Jennifer L. Whitten (Tulane Univ.), Thomas Widemann (Paris Observatory)

A list of all co-signers can be found at https://docs.google.com/spreadsheets/d/1TZGokHreJ3_oP77mTeaj8oVTUY9sO6tvmKqCc537nEc/edit?u $\mathrm{sp}=$ sharing 


\section{Introduction}

Conventional remote sensing of the surface of Venus has long been considered to be impossible due to the thick, $\mathrm{CO}_{2}$-rich atmosphere. A serendipitous observation of Venus's nightside by Allen and Crawford (1984) revealed cloud structures deep within the atmosphere some $20 \mathrm{~km}$ underneath the high-altitude hazes observable at $1 \mu \mathrm{m}$. Follow-up modeling and ground-based observations showed that it is possible to observe the surface through transparent windows in the $\mathrm{CO}_{2}$ spectrum near $1 \mu \mathrm{m}$ (e.g., Kamp et al., 1988). The Galileo (Carlson et al., 1993) and Cassini (Baines et al., 2000) missions used the $1 \mu \mathrm{m}$ windows for remote sensing method during flybys. The VIRTIS and VMC instruments on the ESA mission Venus Express (VEX) was the first instrument to routinely map the surface of Venus using the near-infrared windows from orbit (Mueller et al., 2008; Helbert et al., 2008), followed by brief observations of the IR1 camera on the JAXA Akatsuki mission (Nakamura et al., 2016).

In response to these successes, the Venus Emissivity Mapper (VEM) concept was developed for future orbital missions to enable Venus' surface to be studied from orbit through six different windows at 0.86, 0.91, 0.99, 1.02, 1.11, and $1.18 \mu \mathrm{m}$ (Helbert et al., 2018, 2019). It is proposed as part of the VERITAS (Helbert et al., 2018; Smrekar et al., 2020) and Envision (Helbert et al., 2019) missions. In a manner analogous to the eight-filter imaging on the Pancam instrument of the Mars Exploration rovers (Bell et al., 2003) or the imaging of the surface of Titan by the VIMS instrument on the Cassini mission (Barnes et al, 2007), the six windows can provide a wealth of information on surface composition. In the case of Venus, the windows occur conveniently in a diagnostic spectral region between most $\mathrm{Fe}^{3+}$ and $\mathrm{Fe}^{2+}$ features in silicates. Thus, they have the potential to provide great insights into Venus surface geology.

This white paper describes how orbital spectra can be measured and calibrated using windows in the $\sim 1 \mu \mathrm{m}$ region where the Venus atmosphere is transparent. It discusses how previous pilot studies of the VIRTIS orbiter produced exciting science results with only a single measurement band at $1.02 \mu \mathrm{m}$. It explains trends seen in laboratory emissivity measurements that can be used to interpret orbital data. It highlights the tremendous potential of using six-band orbital emissivity measurements to resolve decades-old questions by providing quantitative data to interpret Venus surface rock types. Selection of a VEM-like instrument for flight on any Venus orbital mission would lead to a highly accurate global map of Fe content and inferred rock type if accompanied by accurate topographic data from an accompanying radar optimized for high precision DEM measurements such as VISAR (Hensley et al., 2015). The combination of orbital spectroscopy and a robust $480^{\circ} \mathrm{C}$ laboratory calibration suite will poise Venus science for a dramatic improvement in understanding of surface geology and the formation and internal processes of Earth's twin planet.

\section{VIRTIS observations of the surface of Venus}

Venus Express VIRTIS results not only provided proof of concept results for orbital spectroscopy, but also produced outstanding science using primarily only one emissivity band at $1.02 \mu \mathrm{m}$. Helbert et al. (2008) and Mueller et al. (2008) provided the first indication of a systematic correlation between emissivity variations and geological units from the VIRTIS data. Higher emissivity was seen for younger volcanic features and lower emissivity for the oldest tesserae units. Smrekar et al. (2010) showed that Venus was volcanically active in the recent geological past. Gilmore et al. (2015) demonstrated that the emissivity anomaly on the tesserae is not an artifact of the uncertainty in the topography but rather indicative of a difference in surface composition consistent with lower iron content and the interpretation that tessera may be continental analogs. Others (e.g., D’Incecco et al., 2017 and Stofan et al., 2016) conducted 
regional studies of volcanic landforms. Mueller et al. (2012) discussed the limits on the rate of volcanic activity from VIRTIS on Venus Express and potential improvements to be gained through use of an instrument like VEM. Gilmore et al. (2017) placed Venus Express results and recent laboratory work in the general context of the surface composition of Venus. Considering the significance of these studies based on one band, it is clear that spectroscopic investigations using emissivity bands truly are mature and ready for the additional exciting science to be gained by use of a broader wavelength range.

\section{Emissivity measurements through the Venus atmosphere}

VIRTIS on Venus Express was not built for surface observations. In fact, the $1 \mu \mathrm{m}$ region was at the very edge of the sensitivity of the IR detector. This combined with thermal drifts resulting in drifts of the wavelength calibration and straylight issues resulted in considerable uncertainties in the measurements. This raises the question how well a dedicated instrument could perform Venus surface spectroscopy. To assess the uncertainties associated with orbital measurements, we use here as an example instrument the Venus Emissivity Mapper (VEM), which was designed and optimized specifically for this type of observation. It uses 14 bands to probe various atmospheric windows (Figure 1) as measured by night-side emission data from the SPICAV instrument on Venus Express (Korablev et al., 2012, Marcq et al. 2017). There are five useful windows for surface measurements, one of which (ca. 0.96$1.04 \mu \mathrm{m})$ is wide enough to accommodate two band filters. Eight additional channels measure atmospheric water vapor abundance, clouds, and stray light as needed to correct for the atmospheric contribution to the surface rock data (Helbert et al., 2018).

In brief, the error analysis uses an

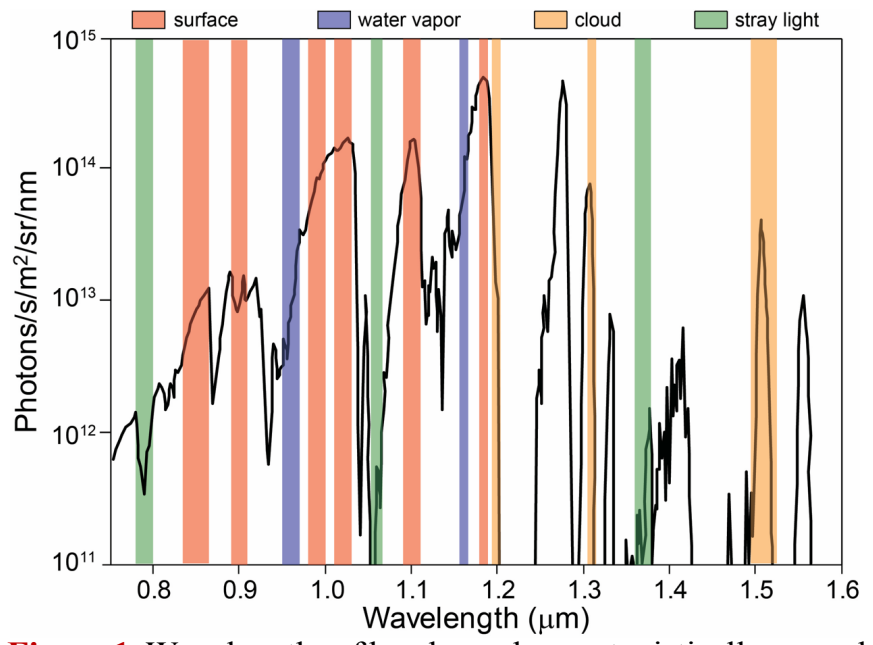

Figure 1. Wavelengths of bands used opportunistically around gaps in the $\mathrm{CO}_{2}$ atmosphere of Venus. Collectively, these offer a comprehensive sampling of surface, water vapor cloud opacity, and stray light as needed to estimate errors on surface bands. Black line is an observed night-side emission spectrum of Venus. Adapted from Helbert et al. (2018).

update to the extensively tested data

processing pipeline developed to process VIRTIS surface data (Müller et al., 2008), combined with a radiative transfer model (RTM) (Haus et al., 2016; Kappel et al., 2015, 2016, Müller et al 2020). Ground processing would be done at $10 \mathrm{~km}$ spatial resolution. The RTM estimates and bounds uncertainties introduced by atmospheric variables, including cloud-droplet $\mathrm{H}_{2} \mathrm{SO}_{4}$ concentrations, cloud layer top and bottom altitudes, and particle-size distribution for the distinct size modes termed $1\left(\mathrm{r}_{\mathrm{eff}}=0.30 \mu \mathrm{m}\right), 2\left(\mathrm{r}_{\mathrm{eff}}=1.0 \mu \mathrm{m}\right), 2^{\prime}\left(\mathrm{r}_{\mathrm{eff}}=1.40 \mu \mathrm{m}\right)$, and $3\left(\mathrm{r}_{\mathrm{eff}}=3.65 \mu \mathrm{m}\right)$ in Venus' clouds (Barstow et al., 2012; Knollenberg and Hunten, 1980, Haus et al. 2013).

The RTM uses a set of atmospheric parameters derived from fitting a VEX SPICAV spectrum from 0.75 to $1.60 \mu \mathrm{m}$ (Korablev et al., 2012). The six VEM surface bands are fitted in the RTM by varying surface emissivity as a free parameter, using cloud-column densities derived from three VEM cloud opacity channels, as well as two VEM water vapor channels and surface temperatures derived from a digital elevation model (DEM)(Figure 1). The DEM assumes use of 
Table 1. Uncertainties in Emissivity Retrieval

\begin{tabular}{|c|c|c|c|c|c|c|c|}
\hline \multicolumn{2}{|c|}{ Surface band center $\lambda(\mu \mathrm{m})$} & 0.86 & 0.91 & 0.99 & 1.02 & 1.10 & 1.18 \\
\hline Error source & $\begin{array}{c}\text { Assumed } \\
\text { uncertainty }\end{array}$ & \multicolumn{6}{|c|}{ Single look emissivity uncertainty from various errors sources $(\%)$} \\
\hline Cloud mode 1 & $25 \%$ & 1.17 & 1.23 & 0.41 & 0.24 & 0.25 & 0.71 \\
\hline Cloud mode 2 & Retrieved & \multicolumn{6}{|c|}{ See below, derived from $\mathrm{I}_{\mathrm{atm}}$} \\
\hline Cloud mode 2' & $25 \%$ & 1.49 & 1.65 & 0.66 & 0.40 & 0.26 & 0.79 \\
\hline Cloud mode 3 & Retrieved & \multicolumn{6}{|c|}{ See below, derived from $\mathrm{I}_{\mathrm{atm}}$} \\
\hline Cloud mode 1 top altitude & $1.5 \mathrm{~km}$ & 0.01 & 0.01 & 0.01 & 0.01 & 0.01 & 0.03 \\
\hline Cloud mode 2 top altitude & $1.5 \mathrm{~km}$ & 0.02 & 0.02 & 0.02 & 0.02 & 0.00 & 0.09 \\
\hline Cloud mode 2' top altitude & $1.5 \mathrm{~km}$ & 0.00 & 0.03 & 0.03 & 0.03 & 0.08 & 0.29 \\
\hline Cloud mode 3 top altitude & $1.5 \mathrm{~km}$ & 0.01 & 0.07 & 0.04 & 0.04 & 0.15 & 0.66 \\
\hline Cloud base altitude & $0.5 \mathrm{~km}$ & 0.01 & 0.16 & 0.12 & 0.13 & 0.36 & 1.62 \\
\hline $\mathrm{H}_{2} \mathrm{SO}_{4}$ conc. & $3.75 \%$ & 0.43 & 0.58 & 0.4 & 0.2 & 0.07 & 1.37 \\
\hline Atmospheric error $\Delta \varepsilon_{\mathrm{atm}}(\%)$ & $\begin{array}{c}\text { Root sum of } \\
\text { squares of } \\
\text { column above } \\
\end{array}$ & 1.943 & 2.146 & 0.884 & 0.527 & 0.542 & 2.482 \\
\hline & & \multicolumn{6}{|c|}{ Calculation of observational uncertainty } \\
\hline \multirow{2}{*}{ Stacked Images $\mathrm{N}=18$} & \multirow{2}{*}{$\begin{array}{c}\text { Binned Pixels } \\
\mathrm{M}=4\end{array}$} & \multicolumn{6}{|c|}{ Surface band radiance measurement uncertainty per pixel $\Delta \mathrm{I}_{\text {surf }}(\%)$} \\
\hline & & 0.442 & 0.236 & 0.053 & 0.053 & 0.054 & 0.031 \\
\hline Obs. Error & \multicolumn{7}{|c|}{ Relative partial derivatives of derived emissivity with respect to observables } \\
\hline$\Delta \mathrm{c}_{\mathrm{H} 2 \mathrm{O}}=0.3 \%$ & $\mathrm{~d} \varepsilon / \mathrm{dc}_{\mathrm{H} 2 \mathrm{O}}$ & 0.114 & 1.486 & 0.629 & 0.042 & 5.795 & 4.773 \\
\hline$\Delta \mathrm{I}_{\text {cloud }}=0.3 \%$ & $\mathrm{~d} \varepsilon / \mathrm{dI}_{\text {cloud }}$ & 2.472 & 3.704 & 2.658 & 2.370 & 13.027 & 12.526 \\
\hline See above & $\mathrm{d} \varepsilon / \mathrm{dI}_{\text {surf }}$ & 3.384 & 4.301 & 3.188 & 2.967 & 13.716 & 13.098 \\
\hline$\Delta \mathrm{Z}=5 \mathrm{~m}$ & $\mathrm{~d} \varepsilon / \mathrm{dZ}[\% / \mathrm{m}]$ & 0.060 & 0.060 & 0.050 & 0.048 & 0.103 & 0.098 \\
\hline $\begin{array}{c}\text { Total emissivity uncertainty } \\
\Delta \varepsilon(\%) \\
\end{array}$ & $\Delta \varepsilon$ & 0.704 & 0.752 & 0.386 & 0.310 & 0.848 & 1.082 \\
\hline
\end{tabular}

a Venus synthetic aperture radar (VISAR) to provide topography with a mean height accuracy of $5 \mathrm{~m}$ or better and a spatial resolution of $10 \mathrm{~km}$ or better.

The three VEM cloud bands permit accurate accounting of varying cloud opacity. High optical depth of $\tau>20$ (Haus et al., 2013) of the atmosphere results in intense multiple scattering, removing directional information. Retrieval for particle properties using varying viewing geometries as done at Mars and Titan is not possible at Venus. Instead, the effect of lower-atmosphere temperature uncertainty is removed statistically (Kappel et al., 2016, Stofan et al., 2016) and controlled for by comparing areas of similar average elevation (Müller et al., 2020). This is possible because the lower atmosphere efficiently redistributes heat (Stone, 1975), as confirmed by a Venus global circulation model (Lebonnois et al., 2018), showing that temperatures vary little over time and horizontal distance. Reproducibility of VIRTIS emissivity retrievals in Kappel et al. (2016) over different local times is consistent with these small temperature differences (Stone, 1975; Lebonnois et al., 2018). Analysis of VIRTIS data bounds the atmospheric influence. The $2 \sigma$ residual atmospheric errors cited from Kappel et al. (2015) are calculated for emissivity retrieval from a single VIRTIS spectrum. However, because VEM observes each spot on the surface multiple times, short-term (spatially and temporally) variability of the atmosphere is reduced by averaging image swaths acquired at different times.

Table 1 shows estimated errors considering predicted atmospheric variability and expected instrument performance. It quantifies three groups of uncertainties of the atmospheric parameters going into:

1) the emissivity error due to VEM measurement uncertainty of the retrieved water vapor abundance $\Delta \varepsilon_{H_{2} O}$, cloud opacity $\Delta \varepsilon_{\text {cloud, }}$, and surface window radiance $\Delta \varepsilon_{\text {surf }}$;

2) short term-variability $\Delta \varepsilon_{\text {atm }}$ of the atmosphere not constrained by observations, and

3 ) uncertainties in the surface topography, $\Delta \varepsilon_{Z}$. 
The first component can be improved statistically by the total number of looks (MN) of the same surface element. The second component can be statistically improved by the number of overlapping looks $(\mathrm{N})$ on adjacent orbits. The third component cannot be statistically improved. The total resultant error on each channel is given in the $2^{\text {nd }}$ to bottom row of Table 1 . Capability for emissivity precision is better than $1.09 \%$ for all bands.

The final step in generating orbital emissivity data is to convert the results from relative emissivity to absolute. This process utilizes the same methodology employed for interpretation of data from the Moon (Wu et al., 2018). For Venus, we have three landing sites where Soviet landers produced suites of geochemical analyses. Orbital coverage should be planned to cover all three of these regions to provide optimal possibilities for creating a spectral calibration correction factor to relate laboratory absolute emissivity to relative emissivity from orbit.

\section{Interpreting Venus orbital spectra based on laboratory measurements}

VIRTIS data were

interpreted on the basis of relative emissivity changes. But six-band orbital data and coverage of landing sites for calibration will provide results that can be interpreted using absolute measurements from laboratory data. For this purpose, a unique Venus setup was created at the Planetary Spectroscopy Laboratory (PSL) at DLR for the study of the emissivity of Venus-analog materials at Venus surface temperatures. PSL has several external simulation chambers attached to Bruker Vertex 80V FTIR spectrometers to measure the emissivity of solid and granular samples. One chamber features a highefficiency induction system to heat the samples under vacuum to temperatures from $320 \mathrm{~K}$ up to above $900 \mathrm{~K}$, while keeping the chamber at almost ambient temperature. A shutter
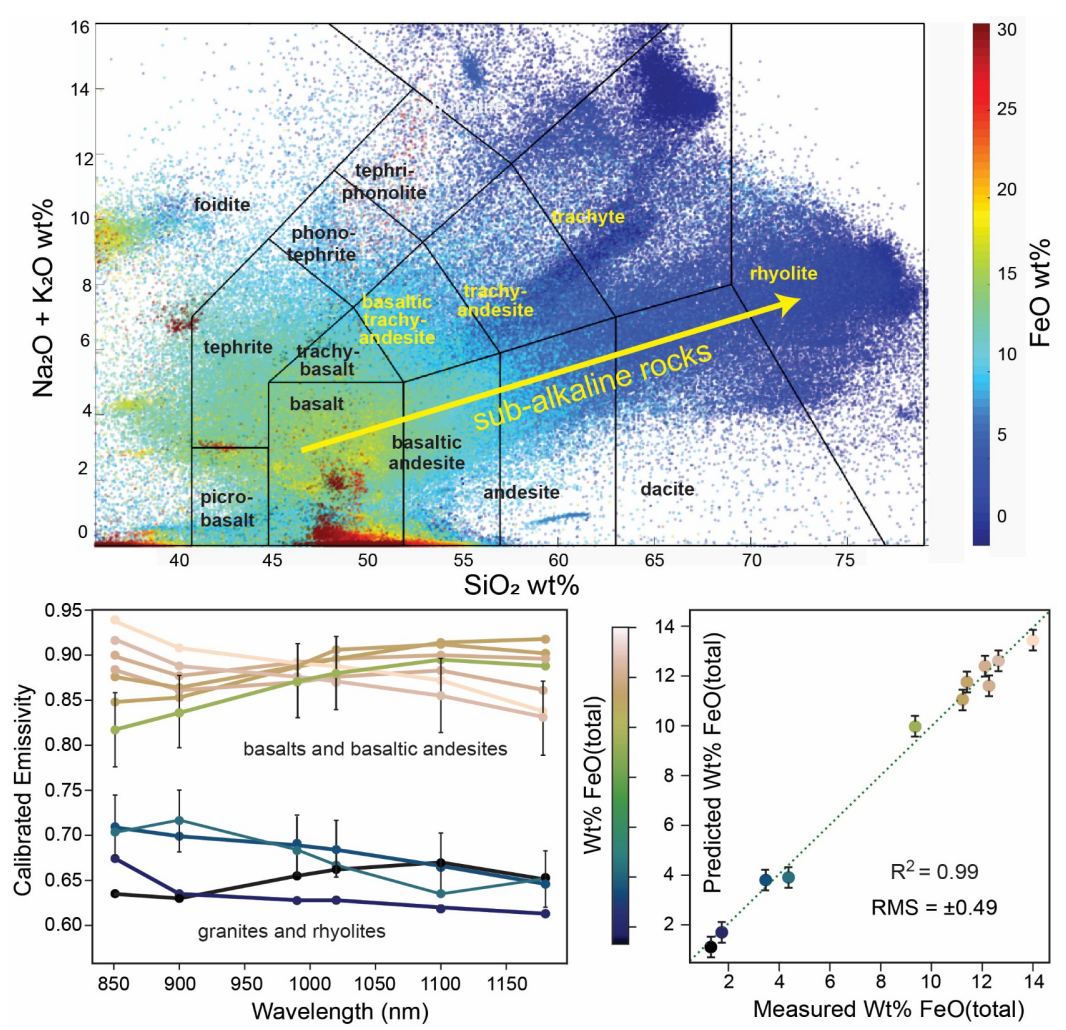

Figure 2. (top) Chemical compositions of terrestrial volcanic rocks plotted on a total alkali vs. $\mathrm{SiO}_{2}$ diagram. Wt $\% \mathrm{FeO}$ contents of 486,629 global nonmarine volcanic (GeoRoc Database, http://georoc.mpchmainz.gwdg.de/georoc/, accessed 6/24/20) and 26,879 oceanic rocks (PetDB Database, (www.earthchem.org/petdb; accessed 3/7/20). Plutonic rocks in GeoRoc $(105,448$ rocks) follow nearly-identical trends. These data show that $94 \%$ of terrestrial igneous rocks lie along the sub-alkaline trend from picrobasalt to rhyolite, so rock standards along this trend were measured in the lab to build predictive equations for Venus. (bottom left) $480^{\circ} \mathrm{C}$ laboratory spectra show distinct differences between basalts and basaltic andesites vs. granites/rhyolites far greater than the required $4 \%$ errors shown (on only two spectra for clarity). Current best estimate errors including instrument performance and atmospheric uncertainties are significantly smaller (Table 1). (bottom right) Partial least squares regression modeling of the six VEM channels yields excellent agreement between predicted and true $\mathrm{FeO}$ contents with errors of $\pm 0.49 \mathrm{wt} \% \mathrm{FeO}$. Adapted from Dyar et al. (submitted, a). 
allows for separating the spectrometer from the external chamber. An optical window (vacuum tight) can be mounted at the entrance of the emissivity chamber to keep the external chamber at $\geq$ ambient pressure, under purged air or inert gases. Ceramic cups enclosing a stainless steel disc have elevated rims enclosing the samples to heat it from all sides, effectively suppressing thermal gradients. Several temperature sensors in the emissivity chamber measure the sample temperature as well as monitoring the range of equipment and chamber temperatures. A webcam mounted in the emissivity chamber monitors the heated sample and its vicinity.

In choosing standards for lab measurements, it was recognized that the Venus surface is quite dissimilar from those of the Moon and Mars because of the paucity of sediment or regolith. The few sparse particulate sediments are impact-derived and occur primarily in two dune fields $\left(\sim 18,410 \mathrm{~km}^{2}\right.$; Bourke et al., 2010), yardangs (40,000 km²; Greeley et al., 1995), wind streaks (1.3 million $\mathrm{km}^{2}$; Greeley et al., 1994), and extended ejecta blankets (40 million $\mathrm{km}^{2}$; Campbell et al., 1992). Collectively these features account for a very small part ( $9 \%)$ of the 460 million $\mathrm{km}^{2}$ of the Venus surface. Although higher resolution imagery may eventually increase the scales of our inventory of particulates on Venus, it is apparent that sedimentary systems on Venus are supply-limited by the dearth of impacts. Thus, sample selection for Venus-analog studies was biased toward slabbed samples, though further work on particulate samples is planned.

PSL laboratory data from 11 sub-alkaline rocks are plotted in Figure 2 (bottom left). It is immediately apparent that emissivity of Fe-rich basaltic rocks is much higher than that of compositionally equivalent low-Fe, high $\mathrm{Si}$ granites and rhyolites. Thus, data from an orbiter with six bands in this region will immediately resolve the question of the rock type of tessera. But it is likely that even more subtle distinctions can be made using such data, as explained in Dyar et al., submitted, a).

Igneous rocks are typically classified on the basis of chemical information about $\mathrm{Na}, \mathrm{K}$, and $\mathrm{Si}$ (e.g., the total alkali vs. silica TAS diagram for volcanic rocks at the top of Figure 2). Because those elements are featureless in the $1 \mu \mathrm{m}$ region, orbital identifications of Venus rock types instead depend upon transition metals (dominantly Fe) that do have spectral features in that region. The challenge, then, is to relate $\mathrm{FeO}$ contents to rock classification. So, Figure 2 (top) shows the distribution of $\mathrm{FeO}$ contents contoured onto the TAS plot. $\mathrm{FeO}$ varies from basalt to rhyolite, and therefore can be used as a proxy for rock type. Table 2 gives average FeO contents for different rock types in the TAS classification.

Given this trend, it should be possible to use $\mathrm{FeO}$ contents derived from emissivity spectra to predict rock type. A partial least squares (PLS) regression model was created using the 11 sub-alkaline rock standard spectra shown in Figure 2 (bottom left). RMSE values for that PLS model indicate that $\mathrm{wt} \% \mathrm{FeO}$ (total) can be predicted with an accuracy of \pm 0.49 as seen in the

Table 2. Wt\% FeO (total) Contents of Volcanic Rock Types*

\begin{tabular}{lrrrlrrr}
\hline Name & Average & StDev & \multicolumn{1}{c}{$\#$} & Name & Average & StDev & $\#$ \\
\hline Picrobasalt & 9.50 & 4.18 & 13,163 & Trachyte & 2.29 & 2.27 & 25,928 \\
Basalt & 9.86 & 5.68 & 156,533 & Trachyandesite & 3.68 & 3.15 & 18,854 \\
Basaltic andesite & 8.69 & 5.87 & 65377 & Tephrite & 11.55 & 3.27 & 19,169 \\
Andesite & 6.47 & 1.95 & 22,378 & Phonotephrite & 9.72 & 6.20 & 2,977 \\
Dacite & 4.31 & 1.71 & 17,465 & Tephriphonolite & 7.47 & 6.35 & 2,286 \\
Rhyolite & 2.19 & 1.43 & 28,944 & Phonolite & 3.37 & 2.48 & 4,374 \\
Trachybasalt & 10.39 & 2.63 & 12,187 & Foidite & 25.95 & 22.35 & 142,765 \\
Basaltic trachyandesite & 6.52 & 4.13 & 16,002 & & & \\
\hline *Based on all 486,629 global non-marine volcanic rocks in the GeoRoc Database, http://georoc.mpch- & \\
mainz.gwdg.de/georoc/, accessed 6/24/20) and 26,879 marine rocks (PetDB Database, \\
www.earthchem.org/petdb; accessed 3/7/20). Plutonic rocks (105,448 rocks, not shown) follow similar rends.
\end{tabular}


plot of predicted vs. measured $\mathrm{FeO}$ content in Figure 2 (bottom right). This difference is more than sufficient to discriminate between basaltic and rhyolite/granitic rocks as well as intermediate rock types along the trend. For example, consider the problem of distinguishing the two possibilities for tessera compositions: basalt $(\bar{x}=9.86 \mathrm{wt} \% \mathrm{FeO}$ from Table 2) vs. granite/rhyolite $(\bar{x}=2.19 \mathrm{wt} \% \mathrm{FeO})$. For most of the Venus surface, where it is safe to assume sub-alkaline magmatism, distinctions indicative of geologic origin would be easily identifiable. With \pm 0.49 prediction accuracy from lab data and the $4 \%$ error on the orbital measurement, even the intermediate rock types between basalt and rhyolite might be distinguished: basaltic andesite $(\bar{x}=8.69 \mathrm{wt} \% \mathrm{FeO})$ andesite $(\bar{x}=6.50 \mathrm{wt} \% \mathrm{FeO})$, and dacite $(\bar{x}=4.31 \mathrm{wt} \% \mathrm{FeO})$. With CBE uncertainties, the values would be even more distinct. As the spectral library on which these data are based increases in size, the errors should continue to decrease, and the ability of our models to distinguish among the two populations should improve.

It is also possible that more alkaline rocks might exist on the Venus surface, as suggested by Venera 13 geochemistry (Barsukov et al., 1982) based on a single $\mathrm{K}_{2} \mathrm{O}$ analysis of $4.0 \pm 0.63$ wt $\%$. The presence of significant alkaline magmas with those compositions is unlikely on Venus given the large volumes of melting that caused the prominent pervasive volcanic flows. To assess this possibility, a model was also constructed from the seven samples that are phonolitic and tephritic. These are predicted separately because rocks rich in alkalis differ from normal igneous counterparts due to structural changes in their parent melts. Structural differences between sub-alkaline and alkaline rocks justify predicting those rock trends separately. So a different algorithm was trained for these seven samples, with an accuracy of $\pm 0.43 \mathrm{wt} \% \mathrm{FeO}$.

\section{Discussion and Conclusions}

Because these are only first results on rocks at Venus temperatures, we do not yet have enough data to begin to parse out effects known to affect remote-sensed spectra, which include grain size, mineralogy, weathering, etc. However, even our small data set provides hints of what further studies may uncover. For example, three samples in this study were run as particulates: a sea sand, basalt (Hawaii fresh) and a granite (Maine), but the igneous rock particulates closely matched their compositional equivalents that were run as slabs. This suggests that mineral/particle grain size effects may be small second order effects in this wavelength range, though further work is needed to explore this.

Some variations in textures were also studied. Surface roughness of sample slabs used here was quite variable because they resulted from rough cuts with different types of rock saws. Three samples were highly porous. Model performance did not improve when such outlier samples were removed from the training set.

Other workers have proposed that Venus surface spectroscopy may be affected by the presence of spectrally impenetrable surface coatings due to weathering that obscure the underlying rock types. However, experiments supporting creation of hematite coatings were done in air and at temperatures too high to be appropriate to Venus (e.g., Filiberto et al., 2019). Single scatter albedo models and analysis of kinetic diffusion data by Dyar et al. (submitted, b) as well as experimental results by Berger et al. (2019) suggest that in fact, the predominant coatings on Venus rocks are a mixture of relatively transparent sulfate (anhydrite) (Zolotov, 2019) and metastable carbonate. So atmospheric interactions are unlikely to cause significant changes in emissivity that could give rise to erroneous interpretations of $\mathrm{FeO}$ content. This conclusion is supported by the strong match between Venera data and the laboratory slab data (Helbert et al., submitted). In short, while we recognize and plan to explicitly test the effects of 
surface texture, porosity, and grain size in future detailed studies, the first set of laboratory results shows great promise even using the empirical PLS regression approach.

Overall, six-band spectroscopy shows great potential for study of the Venus surface. Selection of an instrument with a VEM-like concept for flight on any Venus orbital mission would lead to a global map of Fe content and inferred rock type if accompanied by accurate topographic data from an accompanying radar optimized for high precision DEM measurements such as VISAR (Hensley et al., 2015). The combination of six-band spectroscopy and a robust $480^{\circ} \mathrm{C}$ laboratory calibration suite will poise Venus science for a dramatic improvement in understanding of surface geology and the formation and internal processes of Earth's twin planet.

\section{References}

Allen, D.A. and Crawford, J.W. (1984) Nature, DOI 10.1038/307222a0.

D'Incecco et al. (2017) Planetary and Space Sciences, 136, 25-33. https://doi.org/10.1016/j.pss.2016.12.002.

Baines, K.H. et al. (2000) Icarus, DOI 10.1006/icar.2000.6519 (2000)

Barnes, J.W. et al. (2007) Journal of Geophysical Research (Planets), 112, E11006.

Barstow, J.K. et al. (2012) Icarus, 217(2), 542-560. doi:10.1016/j.icarus.2011.05.018.

Basilevsky, A.T. et al. (2012) Icarus, 217, 434, 10.1016/j.icarus.2011.11.003.

Barsukov, V.L. et al. (1982) Geokhimiya, 1982(7), 899-919.

Bell, J.F. III et al. (2003) Journal of Geophysical Research, 108, DOIL 10.1029/2003JE002070.

Berger, G. et al. (2019) Icarus, 329, 8-23. doi:10.1016/j.icarus.2019.03.033.

Bourke, M.C. et al. (2010) Geomorphology, 121, 1-14.

Campbell, D.B. et al. (1992) Journal of Geophysical Research Planets, 97, E10, 16249-16277.

Carlson, R.W. et al. (1993) Planetary and Space Science, 10.1016/0032-0633(93)90030-6.

Dyar, M.D. et al. (2020) Probing Venus surface iron contents with six-band VNIR spectroscopy from orbit. Geophysical Research Letters, submitted, a.

Dyar, M.D. et al. (2020) Surface weathering on Venus: Constraints from kinetic, spectroscopic, geochemical data. Icarus, submitted, b. Filiberto, J. et al. (2019) Science Advances, 6, eaax7445. doi:10.1126/sciadv.aax7445.

Gilmore, M. S. et al. (2015) Icarus 254, 350-361. doi:10.1016/j.icarus.2015.04.008.

Gilmore, M.S. et al. (2017) Space Science Reviews, 212, 1511-1540.

Greeley, R. et al. (1995) Icarus, 115, 399-420.

Greeley, R. et al. (1994) Science, 263, 358-361.

Hashimoto, G. et al. (2008) Journal of Geophysical Research (Planets), 113, E00B24, 10.1029/2008JE003134.

Haus, R. et al. (2013) Planetary and Space Science, 89, 77-101. doi:10.1016/j.pss.2013.09.020.

Haus, R. et al. (2016) Icarus, 272, 178-205. doi:10.1016/j.icarus.2016.02.048.

Helbert, J. et al. (2018) Infrared Remote Sensing and Instrumentation XXVI, 10765. doi:10.1117/12.2320112.

Helbert, J. et al. (2008) Geophysical Research Letters, 35, 11201. doi:10.1029/2008GL033609.

Helbert, J. et al. (2019) Proceedings 11128, Infrared Remote Sensing and Instrumentation XXVII; 111284, doi:10.1117/12.2529248.

Helbert et al. (2020) Deriving iron contents from past and future Venus surface spectra with new high temperature laboratory emissivity data. Science Advances, submitted.

Hensley, S. et al. (2015) IEEE 5th Asia-Pacific Conference on Synthetic Aperture Radar (APSAR), 362-366.

Kamp, L.W. et al. (1988) Nature, 336, 360.

Kappel, D. et al. (2016) Icarus, 265, 42-62. doi:10.1016/j.icarus.2015.10.014.

Kappel, D. et al. (2015) Planetary and Space Science, 113-114, 49-65. doi:10.1016/j.pss.2015.01.014.

Knollenberg, R.G., and Hunten, D.M. (1980) Journal of Geophysical Research, 85(A13). doi:10.1029/JA085iA13p08039.

Korablev, O. et al. (2012) Planetary and Space Science, 65, 38-57. doi:10.1016/j.pss.2012.01.002.

Lebonnois, S. et al. (2018) Icarus, 314, 149-158. doi:10.1016/j.icarus.2018.06.006.

Marcq, E. et al. (2017) Space Science Reviews 214(1), article 10. doi: 0.1007/s11214-017-0438-5.

Müller, N.T. et al. (2008) Journal of Geophysical Research Planets, 113, E00B17. doi:10.1029/2008JE003118.

Müller, N.T. et al. (2017) Journal of Geophysical Research (Planets), 122, 1021, 10.1002/2016JE005211.

Müller, N.T. et al. (2020) Icarus, 335, 113400. doi:10.1016/j.icarus.2019.113400.

Nakamura, M. et al. (2016) Earth, Planets and Space, 10.1186/s40623-016-0457-6.

Smrekar, S.E. et al. (2020) Lunar and Planetary Science, 51, Abstract \#1449.

Smrekar, S.E. et al. (2010) Science, 328, 605-608. doi:10.1126/science.1186785.

Stofan, E.R. et al. (2016) Icarus, 271, 375-386. doi:10.1016/j.icarus.2016.01.034.

Stone, P.H. (1975) Journal of Atmospheric Science, 32, 1005-1016.

Wu, Y. et al. (2018) The Astronomical Journal, 155, 213.

Zolotov, M. (2019) Oxford Research Encyclopedia, Planetary Science, Oxford University Press,. doi:10.1093/acrefore/9780190647926.013.146. 